\title{
Characterization of Anti-p54 Monoclonal Antibodies and Their Potential Use for African Swine Fever Virus Diagnosis
}

\author{
Weldu Tesfagaber ${ }^{1,2,+}{ }^{\oplus}$, Lulu Wang ${ }^{1,+}$, Ghebremedhin Tsegay ${ }^{1,2}$, Yibrah Tekle Hagoss ${ }^{1}$, Zhenjiang Zhang ${ }^{1}$, \\ Jiwen Zhang ${ }^{1}$, Haoyue Huangfu ${ }^{1}$, Fei Xi ${ }^{1}$, Fang $\mathrm{Li}^{1}{ }^{1}$, Encheng Sun ${ }^{1}$, Zhigao Bu ${ }^{1, *}$ and Dongming Zhao ${ }^{1, *}$ \\ 1 State Key Laboratory of Veterinary Biotechnology, National High Containment Facilities for Animal Diseases \\ Control and Prevention, Harbin Veterinary Research Institute, Chinese Academy of Agricultural Sciences, \\ Harbin 150069, China; welduvet@gmail.com (W.T.); w1l3118@163.com (L.W.); gherielove84@gmail.com (G.T.); \\ goytomtekle@gmail.com (Y.T.H.); zzj1070303866@163.com (Z.Z.); zjw841380675@outlook.com (J.Z.); \\ haoyue3333@163.com (H.H.); xifei170@163.com (F.X.); lifang01@caas.cn (F.L.); sunencheng@caas.cn (E.S.) \\ 2 Department of Veterinary Science, Hamelmalo Agricultural College, Keren 397, Eritrea \\ * Correspondence: buzhigao@caas.cn (Z.B.); zhaodongming@caas.cn (D.Z.) \\ + Weldu Tesfagaber and Lulu Wang equally contributed to this study.
}

check for

updates

Citation: Tesfagaber, W.; Wang, L.; Tsegay, G.; Hagoss, Y.T.; Zhang, Z.; Zhang, J.; Huangfu, H.; Xi, F.; Li, F.; Sun, E.; et al. Characterization of Anti-p54 Monoclonal Antibodies and Their Potential Use for African Swine Fever Virus Diagnosis. Pathogens 2021, 10, 178. https://doi.org/10.3390/ pathogens10020178

Academic Editor: Tanja Opriessnig

Received: 19 January 2021

Accepted: 2 February 2021

Published: 7 February 2021

Publisher's Note: MDPI stays neutral with regard to jurisdictional claims in published maps and institutional affiliations.

Copyright: (c) 2021 by the authors. Licensee MDPI, Basel, Switzerland. This article is an open access article distributed under the terms and conditions of the Creative Commons Attribution (CC BY) license (https:// creativecommons.org/licenses/by/ $4.0 /)$.

\begin{abstract}
African swine fever (ASF) is a highly lethal hemorrhagic viral disease of domestic pigs caused by African swine fever virus (ASFV). Although a good advance has been made to understand the virus, a safe and effective vaccine against ASFV is still lacking and its eradication solely depends on its early and accurate diagnosis. Thus, improving the available diagnostic assays and adding some validated techniques are useful for a range of serological investigations. The aim of this study was to produce and characterize p54 monoclonal antibodies with an ultimate goal of developing a monoclonal antibody-based enzyme-linked immunosorbent assay (ELISA) for ASFV antibody detection. Five monoclonal antibodies against p54 protein expressed in Escherichia coli was generated and their characterizations were investigated. Furthermore, a competitive enzyme-linked immunosorbent assay (cELISA) based on a monoclonal antibody designated as 2A7 was developed. To evaluate the performance of the assay, a total of 365 pig serum samples (178 negative and 187 positive samples) were tested and a receiver-operating characteristic (ROC) analysis was applied to determine the cut-off value. Based on the ROC analysis, the area under the curve (AUC) was 0.982 (95\% confidence interval: $96.9 \%$ to $99.4 \%$ ), besides a sensitivity of $92.5 \%$ and a specificity of $98.9 \%$ was achieved when the percent inhibition of $20 \%$ was selected as a threshold. Moreover, the result showed an excellent agreement when compared to other commercially available blocking ELISA (kappa value $=0.912$ ) and showed no reaction to other swine pathogens. Overall, the newly developed cELISA method offers a promising approach for a rapid and convenient ASFV serodiagnosis, which could be used as alternative to other serological assays for screening possible ASFV infection.
\end{abstract}

Keywords: African swine fever virus; p54; monoclonal antibodies; competitive ELISA; diagnosis

\section{Introduction}

African swine fever (ASF) is a highly lethal hemorrhagic viral disease of swine that usually results to a mortality rate approaching 100\% in domestic pigs and is classified as a notifiable disease by the World Organization for Animal Health (OIE). Currently, it is the major threat to global pig industry and is caused by African swine fever virus (ASFV), a large and complex double stranded DNA virus with icosahedral morphology [1,2]. Despite of the extensive ongoing research on ASFV, a safe and effective vaccine is still lacking and its control and eradication solely depends on its rapid and accurate diagnosis.

ASFV affects both domestic and wild pigs. However, in domestic pigs there is a variation in the clinical manifestations depending on the virus strains, which differs from an acute, highly lethal hemorrhagic disease to a mild inapparent infection $[3,4]$. On the contrary, it leads to a mild subclinical infection in the natural reservoir's hosts (wart 
hogs and bush pigs), which are the potential source of infection to domestic pigs. It is also important to note that, wild boars (the substantial reservoir of ASFV in Europe and probably also in Asia) show a high susceptibility to ASFV with a disease development similar to domestic pigs [5]. Owing to the importance of the presence of seropositive animals to sub-acute or chronic form of ASFV, there is always a need of an accurate serological diagnosis. Additionally, in the recent years there have been a dramatic increase of ASF outbreak in some Asian and European countries which led to a renewed interest of combating the disease [6-9]. Therefore, to detect sub-acute or chronic nature of ASF either in domestic pigs or the reservoir hosts, a range of sensitive serological investigations are needed and antibody detection is a rational approach.

Numerous ELISA-based serological assays integrating the major capsid protein $\mathrm{p} 72 / \mathrm{B} 646 \mathrm{~L}$, the structural and highly immunogenic protein $\mathrm{p} 30 / \mathrm{CP} 204 \mathrm{~L}$ and polyprotein pp62/CP530R antigens are available for ASFV antibody detection [10-13]. Alternatively, the structural and immunogenic p54/E183L protein is also a highly relevant antigen for serological diagnosis $[11,14,15]$. P54/E183L is a type II transmembrane protein and contains a potential membrane-spanning domain close to the $\mathrm{N}$-terminus [16]. A two-dimensional gel electrophoresis study on ASFV particle, revealed that p54 has a molecular size of about $25 \mathrm{kDa}$ with an isoelectric point of 6.5 [17]. Also, during cell culture adoption and propagation, ASFV generates virus sup-populations differing in p54 molecular size, which may have a role on the mechanism of genetic diversification of ASFV [16,17].

A number of studies have reported that p54 is one of the most important ASFV proteins and plays a key role in virus morphogenesis and viral infection. Anti-p54 sera were found to inhibit ASFV attachment to susceptible cells, suggesting its role in virus entry [18]. Similarly, p54/E183L gene plays an essential role in virus viability [19] and recruitment of envelop precursors to assembly sites [20]. Most importantly, E183L gene is crucial for virus particle transporting to perinuclear factory via direct binding to light chain 8 (LC8) of the dynein [21,22]. A short p54 peptide (149-161 aa) near to its C-terminus is enough to bind to dynein light chain 8 (DLC8) and act as a cargo transport for virus particle [21].

It is proved that, pigs either naturally or experimentally infected with ASFV or inoculated with p54 replicon particle produce high level of anti-p54 antibodies [8,11,15,23-25]. Moreover, antibodies against p54 appear as early as 10 days and persist within the blood of infected animals for several weeks [11,15]. All these characteristics in turn makes p54/E183L gene to be one of the best ASFV antigen targets for development of a serological diagnostic assay. In previous studies, ASFV antibody detection using p54 recombinant protein showed $98 \%$ sensitivity and $97 \%$ specificity on ELISA compared to OIE-ELISA [11] and is a protein of choice for confirmation of ASFV antibody by western blot [14,26]. Although ASFV antibody detection using the most antigenic proteins p72, p30, pp62 together with p54 have been proved to be effective, improving the available diagnostic assays and adding some validated techniques are useful for a range of serological investigations and are a timely demand to mitigate ASFV. Thus, the development of an alternative and more robust diagnostic assay was the focus of this work.

In the current study, a panel of monoclonal antibodies (mAbs) against E. coli expressed p54 recombinant protein were generated and their characterizations were investigated. Further assessing on potential use of these anti-p54 monoclonal antibodies as diagnostic reagent for ASFV antibody detection was performed. Subsequently, a competitive-ELISA (cELISA) based on monoclonal antibody designated as 2A7 was developed. The established competitive ELISA showed high diagnostic sensitivity and specificity for ASFV antibody detection. Overall, the work presented here, provides additional evidence with respect to application of p54 monoclonal antibodies for ASFV diagnosis. 


\section{Results}

\subsection{Expression of p54 Recombinant Protein}

P54/E183L gene of Pig/HLJ/2018 ASFV isolate was expressed as maltose binding protein-tagged fusion protein in Escherichia coli ER2523 strain. The recombinant protein was successfully expressed and following inducing with isopropyl- $\beta$-D-1-thiogalactoside (IPTG), an approximate $65 \mathrm{kDa}$ fusion protein was observed by sodium dodecyl sulfatepolyacrylamide gel electrophoresis (SDS-PAGE) analysis (Figure 1a). Fusion protein was purified using pre-packed MBP Trap column, confirmed by western blot (Figure 1b) and was used as immunogen to immunize BALB/c mice for monoclonal antibody production.

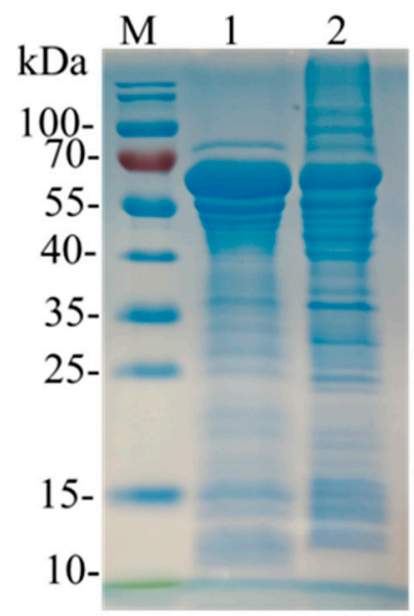

(a)

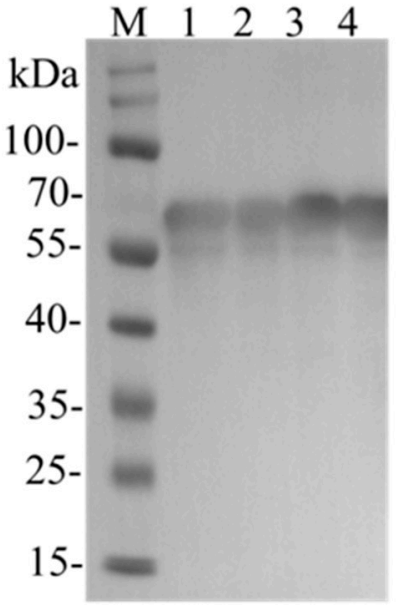

(b)
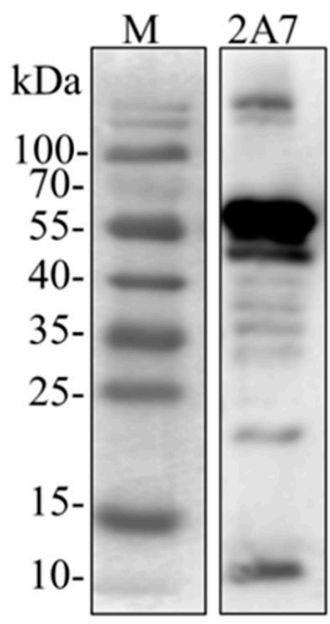
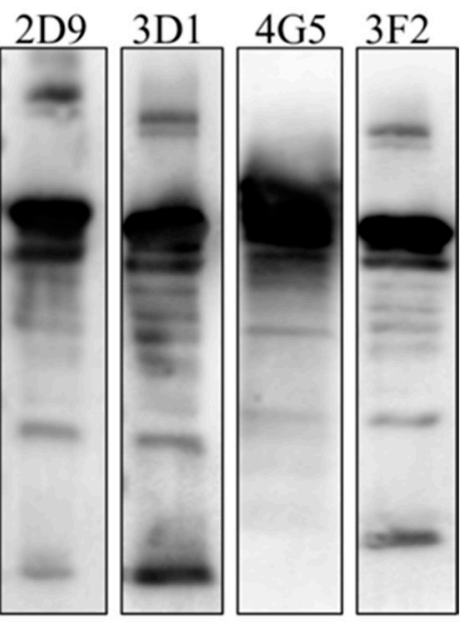

(c)

Figure 1. p54 antigen production and analysis of mAbs. (a): Sodium Dodecyl Sulfate-Polyacrylamide Gel Electrophoresis (SDS-PAGE) analysis of the Maltose Binding Protein (MBP)-tagged p54 recombinant protein expression, followed Coomassie brilliant blue stain. $\mathrm{M}$ is protein marker, lane1 was loaded with $20 \mu \mathrm{L}$ supernatants of bacterial cell lysates after induction with isopropyl- $\beta$-D-1-thiogalactoside (IPTG) and lane2 was loaded with bacterial cell pellets. (b): Western blot analysis of purified p54 recombinant protein using anti-MBP tag antibody. All Lane1 to lane4 were loaded with $10 \mu \mathrm{L}$ purified p54 protein while the left lane is a protein marker. (c): western blot analysis for specificity of mAbs, showing all the five p54 monoclonal antibodies recognize the MBP-p54 protein (about $65 \mathrm{kDa}$ ).

\subsection{Anti-p54 Monoclonal Antibody Production}

We set out to produce a panel of monoclonal antibodies against p54 recombinant protein of African swine fever virus. Following the immunization of BALB/c female mice with p54 recombinant protein, mice with a higher antibody titration to p54 was sacrificed and spleen cells were fused with SP2/0 myeloma cells. Eventually, hybridoma were screened by indirect ELISA and five positive clones specific to p54 designated as 2A7, 2D9, 4G5,3F2 and 3D1 were obtained and subcloned three times by a limiting dilution. Isotypes of monoclonal antibodies were characterized using mouse Ig isotyping kit and all were found to be IgG1 with kappa light chain (Table 1). Further screening by Western blot revealed all monoclonal antibodies were able to recognize an approximate $65 \mathrm{kDa}$ fusion protein (Figure 1c). Similarly, immunofluorescent assay on HEK 293T cells transfected with p54 recombinant gene was performed and all five anti-p54 monoclonal antibodies were able to react, signifying that produced mAbs were specific to p54/E183L gene of ASFV (Figure 2).

Immunofluorescent assay on ASFV infected peripheral alveolar macrophage cells (PAM cells) was also performed and all mAbs were able to detect ASFV infected cells. No labeling was observed in uninfected cells (data not shown). 

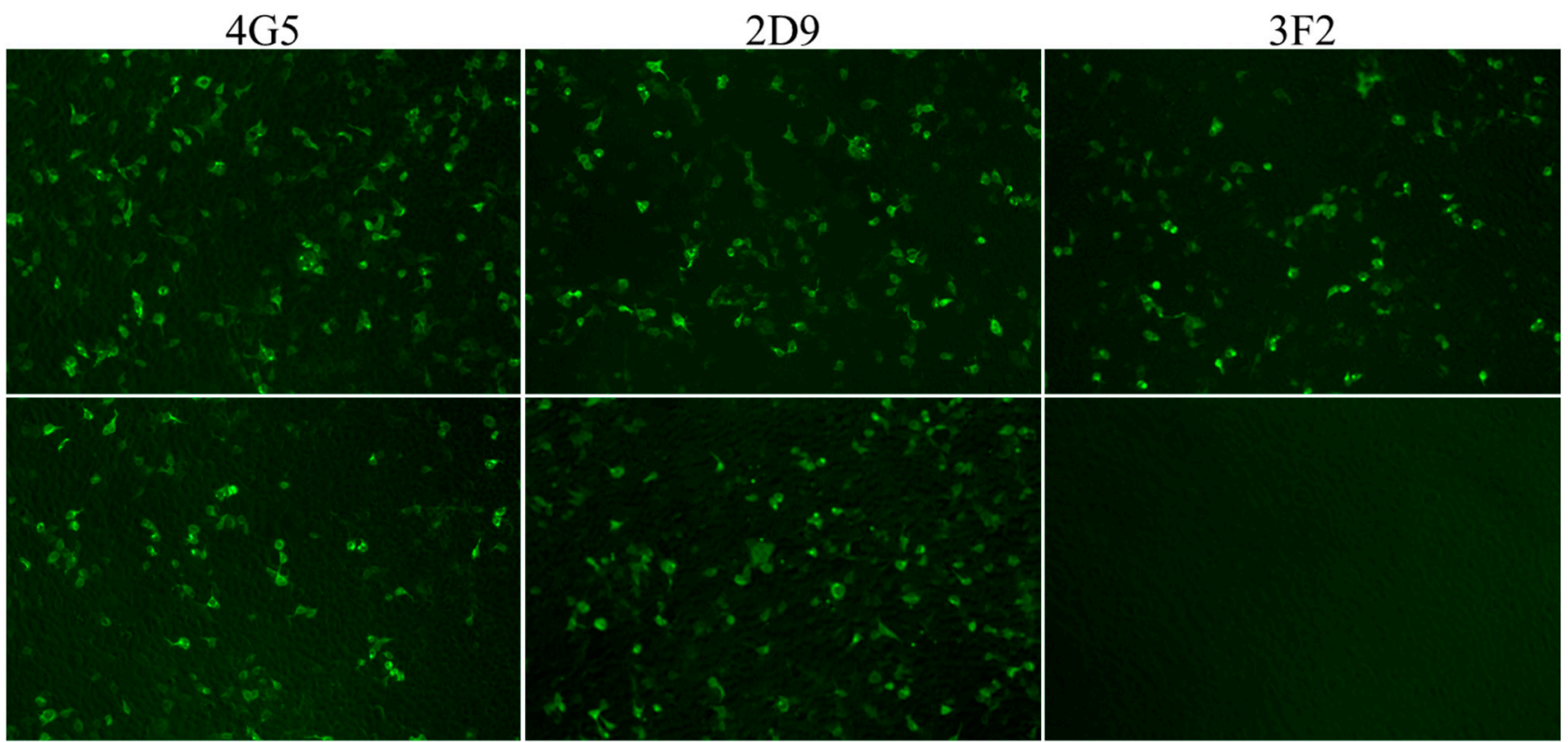

2A7

3D1

Negative

Figure 2. Indirect immunofluorescent assay. HEK 293T cell was transfected with a plasmid expressing viral p54 protein and stained with anti-p54 monoclonal antibodies (4G5, 2D9, 3F2, 2A7 and 3D1) and Alexa Flour 488 FICT- conjugated goat anti-mouse IgG. Following staining all anti-p54 mAbs exhibited green fluorescence. Negative control was HEK 293T cells stained with cell supernatants from SP2/0 mouse myeloma cells.

Table 1. Identification of subclasses of p54 monoclonal antibodies.

\begin{tabular}{cccccc}
\hline & \multicolumn{5}{c}{ Monoclonal Antibodies } \\
\cline { 2 - 6 } & 2A7 & 2D9 & 4G5 & 3F2 & 3D3 \\
\hline Ig subclass & $\operatorname{IgG1}$ & $\mathrm{IgG1}$ & $\mathrm{IgG1}$ & $\mathrm{IgG1}$ & $\mathrm{IgG1}$ \\
Light chain type & $\mathrm{K}$ & $\mathrm{\kappa}$ & $\mathrm{\kappa}$ & $\mathrm{\kappa}$ & $\mathrm{\kappa}$ \\
\hline
\end{tabular}

\subsection{Assessing Potential Uses of p54 Monoclonal Antibodies for Competitive ELISA}

To evaluate the potential use of these anti-p54 monoclonal antibodies as a diagnostic reagent for ASFV antibody detection, competitive-ELISA based on each monoclonal antibody was investigated. Eight ASFV positive serum (from experimentally infected pigs) and four negative serum (from specific-pathogen free pigs) were selected to determine which p54 monoclonal antibody will have a good performance to be applied in competitive ELISA. These samples were nominated on the basis of their OD value on indirect ELISA against p54 recombinant antigen. Each sample was tested with a competitive ELISA at a dilution of 1:10 and the percent of inhibition (PI value) of each sample was calculated. The result found on competitive ELISA revealed that all eight positive test samples were able to block mAb-2A7 by greater than $60 \%$ with a mean PI value of $82 \%$, while for all the negative samples an inhibition percent of less than zero was recorded when mixed to mAb-2A7 (Figure 3-2A7). Therefore, monoclonal antibody 2A7 was selected to develop a competitive ELISA for ASFV antibody detection. 

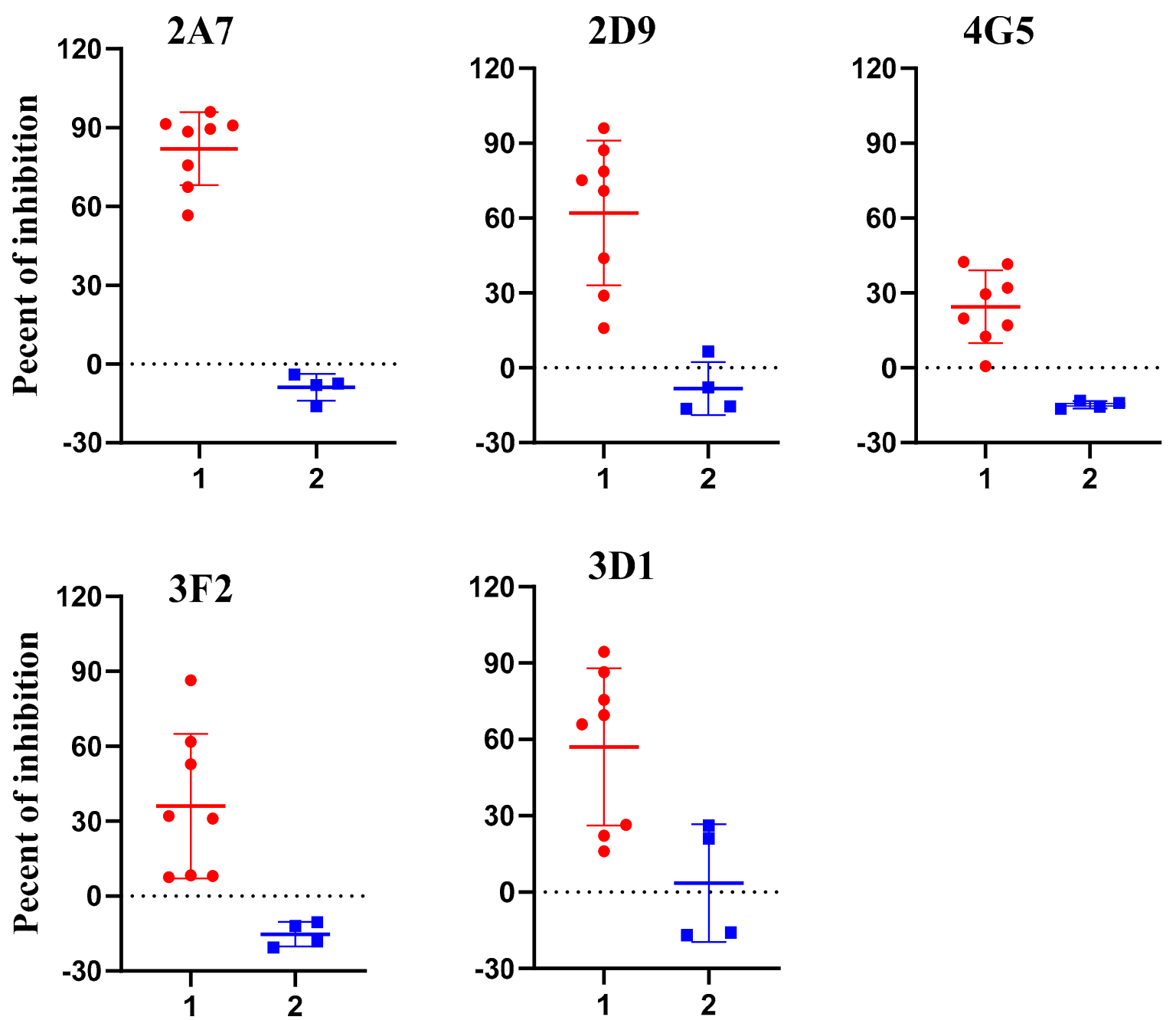

Figure 3. Investigation of p54 monoclonal antibodies on competitive enzyme-linked immunosorbent assay (ELISA) for African swine fever (ASF) detection. In the graph PI value of positive $(n=8)$ and negative $(n=4)$ samples are displayed as dots on two vertical axes $(1=$ positive samples and $2=$ negative samples). All positive sera showed PI value greater than $60 \%$ when competed with p54 monoclonal antibody designated as $2 \mathrm{~A} 7$.

\subsection{Optimization of 2A7-Based Competitive ELISA}

Optimal concentration of coating antigen (p54 recombinant protein), mAb-2A7 and test serum samples were determined by checkboard titration procedure. A concentration of $0.2 \mu \mathrm{g} /$ well of p54 recombinant protein and $1 \mu \mathrm{g} / \mathrm{mL}$ of affinity purified 2A7 were selected as they consistently produce an $\mathrm{OD}_{450}$ value around 1.5 (Figure $4 \mathrm{a}$ ). On the other hand, to optimize serum sample dilution, five known ASFV positive and four known negative serum samples were selected on the basis of their reactiveness to p54 on indirect ELISA. Two-fold serum dilution was tested against a fixed dilution of mAb-2A7 $(1 \mu \mathrm{g} / \mathrm{mL})$ and percent of inhibition was calculated. As shown in Figure $4 \mathrm{~b}$, the dilution rate of serum samples and the PI value of ASFV positive samples were indirectly proportional with a higher PI value recorded at a dilution of 1:10. On the other hand the PI value of negative serum samples were not influenced with serum dilution rate and were below $20 \%$. Based on this data, serum dilution at 1:10 and 1:20 competes well with 2A7 monoclonal antibody and for an optimal inhibition serum dilution was fixed to 1:10 throughout the experiment. 


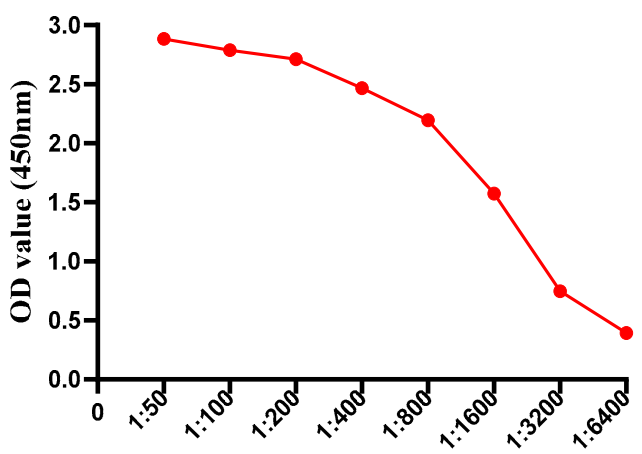

(a)
2A7 dilution

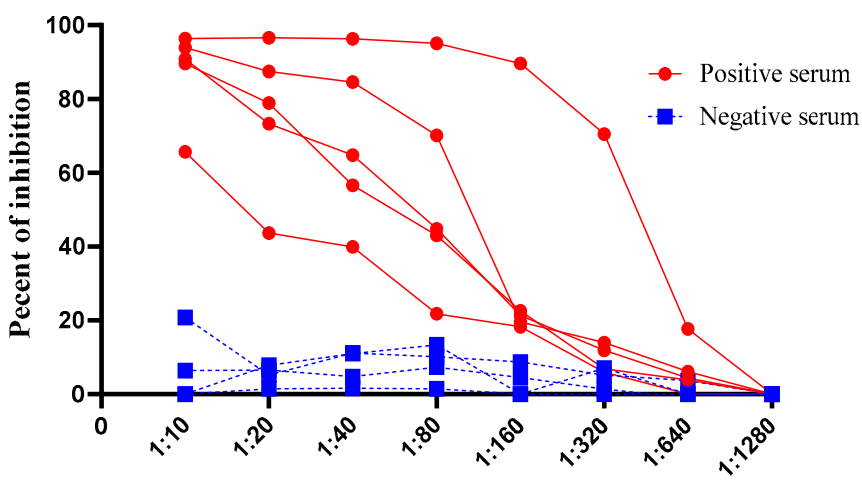

(b)

Serum dilution

Figure 4. Determination of optimal $\mathrm{mAb}-2 \mathrm{~A} 7$ and serum dilution. (a): optimization of monoclonal antibody dilution, $\mathrm{mAb}$ dilution of 1:1000 was chosen as it consistently produces an $\mathrm{OD}_{450}$ value around 1.5. (b): determination of optimal dilution of sera. Serial two-fold dilution of five positive and four negative serum samples were tested against a fixed mAb dilution (1:1000) in competitive enzyme-linked immunosorbent assay (cELISA) and percent of inhibition was recorded. For a better inhibition, serum dilution of 1:10 was selected.

\subsection{Standardization and Determining the Negative Cut-off Value for cELISA}

After optimizing the protocol for competitive ELISA, a total of 365 pig serum samples (178 negative pig serum and 187 positive samples) were tested to evaluate the performance of the assay. These samples were classified as ASFV seronegative or ASFV seropositive according to their known origin and using a commercial ASFV antibody detection kit (Ingezim PPA COMPAC, Ingenasa, Madrid, Spain). All samples were tested in duplicate by the established cELISA and the percent of inhibition value of each sample was calculated. A receiver operating characteristic (ROC) curve statistical analysis was performed and it allowed us to determine the cut-off value and to estimate the diagnostic sensitivity and specificity of the assay (Figure 5b). In addition, an interactive dot plot diagram outlined the PI value of these samples is shown in Figure 5a. Based on the ROC analysis, the area under the curve (AUC) of the established test was 0.982 (95\% confidence interval: $96.9 \%$ to $99.4 \%$ ). Besides, a diagnostic sensitivity of $92.5 \%$ (95\% confidence interval: $87.8 \%$ to $95.9 \%$ ) and a specificity of $98.9 \%$ (95\% confidence interval: $96.0 \%$ to $99.9 \%$ ) were achieved, when the cut-off value was set to $20 \%$. By adopting this cut-off value, out of the 187 ASFV positive samples, 173 were correctly placed as ASFV-seropositive, while only two negative samples were diagnosed incorrectly as false positive (Table 2), indicating that the established cELISA has an excellent diagnostic accuracy.

Table 2. Relative sensitivity and specificity of the newly established cELISA.

\begin{tabular}{cccc}
\hline Ingezim & \multicolumn{2}{c}{ Serum Samples Tested with P54-cELISA } \\
\cline { 2 - 4 } PPA COMPAC Result & Positive & Negative & Total \\
\hline Positive & 173 & 14 & 187 \\
Negative & 2 & 176 & 36 \\
Total & 175 & 190 & 365 \\
\hline
\end{tabular}

Relative sensitivity $=173$ of 187 or $92.5 \%$, relative specificity $=176$ of 178 or $98.9 \%$. 


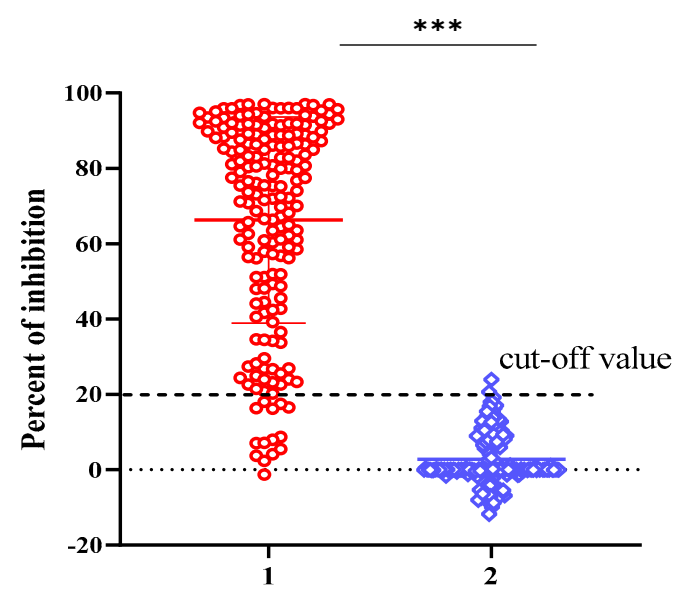

(a)

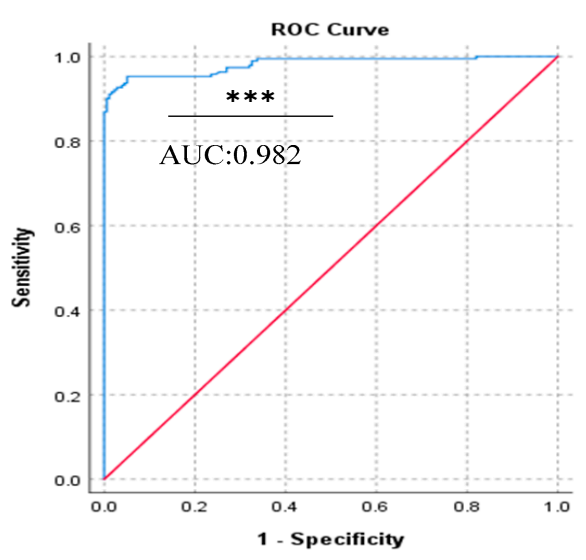

(b)

Figure 5. 2A7-based competitive ELISA analysis of serum samples. The analysis was performed on known ASFV negative samples $(n=178)$ and known ASFV positive samples $(n=187)$. (a): interactive dot plot diagram showing the PI value of pig serum samples and cut-off value was set to $20 \%$. In the graph the PI values of positive and negative samples are displayed as dots on two vertical axes $(1=$ positive samples and $2=$ negative samples $)$. $(\mathbf{b})$ : ROC analysis of 2A7-based cELISA results and the area under the curve (AUC) of the test was 0.982. $p$ value was $<0.0001$ and is indicated by asterisk ${ }^{* * *}$ ).

\subsection{Specificity and Repeatability Test}

To confirm its specificity, the developed cELISA was used to detect four polyclonal anti-sera against other swine viruses. All sera yielded a negative result in the competitive ELISA, with a PI value much lower than the cut-off value (Figure 6). Thus, non-specific positive swine sera were clearly discriminated from the ASFV positive sera, suggesting that the established cELISA has a satisfactory analytical specificity.

To evaluate repeatability of the cELISA, ten randomly picked serum samples (five positive and five negative) were tested by the developed cELISA and the intra and interassay variation was determined by calculating the coefficient of variation $(\mathrm{CV} \%)$. For intra-assay variation, OD values of those ten samples were measured on triplicate on the same plate, while, for inter-assay variation OD values of same samples were measured three times on ELISA plates coated at different times. According to Jacobson [27] an assay with a coefficient of variation (CV) less than $20 \%$ for raw absorbance value is considered to have an adequate repeatability. In this study, the result produced an intra-assay CVs ranging from $0.78 \%$ to $11.27 \%$ and an inter-assay CVs ranging from $5.79 \%$ to $16.8 \%$, showing an excellent repeatability (Table 3 ).

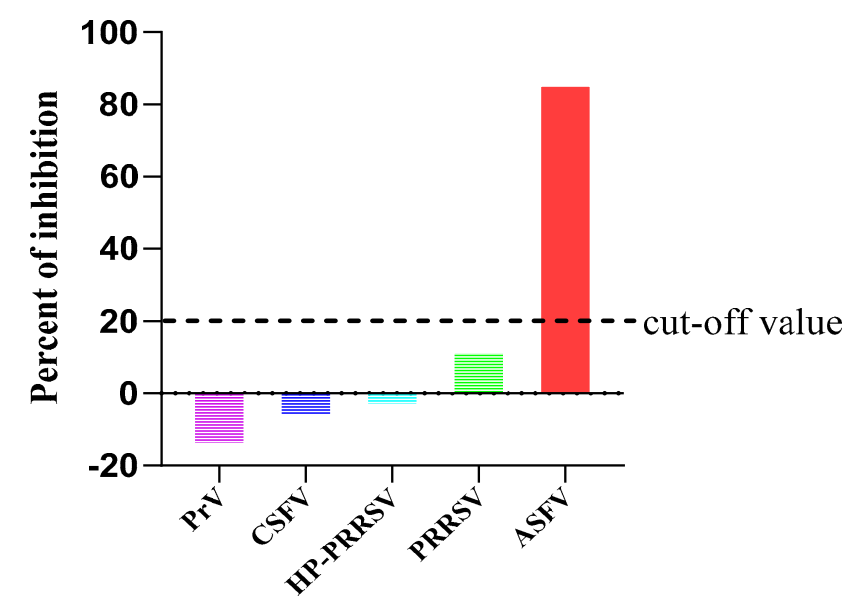

Figure 6. Percent of inhibition of polyclonal anti sera against various porcine viruses after detection with 2A7-cELISA. Only the ASFV positive pig sera had a PI value exceeding the cut-off value. 
Table 3. Intra and inter-assay repeatability of the newly developed cELISA.

\begin{tabular}{ccccccc}
\hline & \multicolumn{3}{c}{ Intra-Assay } & \multicolumn{3}{c}{ Inter-Assay } \\
\cline { 2 - 7 } Samples & $\begin{array}{c}\text { Mean OD } \\
\text { Value }\end{array}$ & SD & CV\% & $\begin{array}{c}\text { Mean OD } \\
\text { Value }\end{array}$ & SD & CV\% \\
\hline Positive 1 & 0.090 & 0.003 & $\mathbf{2 . 7 8}$ & 0.0903 & 0.0103 & $\mathbf{1 1 . 3 6}$ \\
Positive 2 & 0.082 & 0.008 & $\mathbf{9 . 5 2}$ & 0.069 & 0.006 & $\mathbf{8 . 0 7}$ \\
Positive 3 & 0.072 & 0.008 & $\mathbf{1 1 . 2 7}$ & 0.057 & 0.005 & $\mathbf{8 . 7 7}$ \\
Positive 4 & 0.099 & 0.004 & $\mathbf{4 . 0 9}$ & 0.101 & 0.013 & $\mathbf{1 2 . 9 8}$ \\
Positive 5 & 0.150 & 0.004 & $\mathbf{2 . 4 0}$ & 0.137 & 0.023 & $\mathbf{1 6 . 8 0}$ \\
Negative 1 & 0.915 & 0.033 & $\mathbf{3 . 6 0}$ & 0.95 & 0.09 & $\mathbf{9 . 6 7}$ \\
Negative 2 & 0.945 & 0.007 & $\mathbf{0 . 7 8}$ & 0.89 & 0.05 & $\mathbf{5 . 7 9}$ \\
Negative 3 & 0.964 & 0.067 & $\mathbf{6 . 9 2}$ & 0.96 & 0.14 & $\mathbf{1 4 . 0 4}$ \\
Negative 4 & 0.927 & 0.016 & $\mathbf{1 . 6 7}$ & 1.04 & 0.06 & $\mathbf{5 . 9 1}$ \\
Negative 5 & 0.924 & 0.053 & $\mathbf{5 . 7 2}$ & 0.82 & 0.07 & $\mathbf{8 . 7 9}$ \\
\hline
\end{tabular}

\section{Discussion}

In the past 100 years, ASFV has emerged as a major threat to global pig industry. According to the World Organization for Animal Health report on June 2020, the disease is currently circulating in many Asian and European countries, namely, Moldova, Czech Republic, Romania, Hungary, Bulgaria, Belgium, Slovakia, Serbia and Greece from Europe and China, Mongolia, Vietnam, Cambodia, Korea, Laos, Myanmar, Philippines, Indonesia, Papua New Guinea and India from Asia [9]. Equally, it is causing a serious deterioration and incalculable economic impact due to its fast spread.

ASFV virion is a complex, multilayered structure with overall icosahedral morphology and encodes a large number of polypeptides $[1,28]$. Besides, the incomplete understanding of the complex viral structure and its immuno-determinant viral proteins hinders vaccine development against ASFV [29], which has made control and eradication of ASF to depend on applying strict quarantine and biosecurity measures, restriction of animal movements and slaughtering of affected animals [6]. Moreover, to apply these control and eradication measures, an early and accurate diagnosis of ASFV is valuable. On this regard, diagnostic identification of antibodies is a major epidemiological tool used to detect the occurrence of ASFV infection, where clinical signs have not been manifested. For instance, presence of ASFV antibodies implies previous infection and are good markers for disease diagnosis $[11,30]$. Moreover, antibodies against ASFV appears soon after infection and persist for several months to years [31]. Therefore, a sensitive and reliable serological diagnostic assay is required, so as laboratories can effectively and quickly detect ASFV infection. Corresponding to this, identifying potential antigenic ASFV protein targets that suits to develop a diagnostic assay is very important and the structural p54/E183L protein along with p72, p30 and pp62 are among the most.

In the current study, we generated five monoclonal antibodies against the E. coli expressed p54/E183L protein of ASFV and their diagnostic application were evaluated. Analysis of those mAbs with ELISA, western blot and IFA showed that all five monoclonal antibodies were able to recognize the immunizing p54/E183L antigen. The structural p54 protein was fused to MBP in our study and results in a protein with a higher molecular mass about $65 \mathrm{kDa}$, when compared to the actual predicted molecular mass of p54/E183L (25 kDa) [17]. To develop a sensitive and reliable cELISA for ASFV antibody detection, the five mAbs were examined for their ability to compete with polyclonal antibodies from ASFV infected pigs. Accordingly, a monoclonal antibody based-competitive ELISA (2A7-cELISA) was developed. Monoclonal antibody 2A7 was selected, because it demonstrates the strongest competition with ASFV-seropositive samples but not with negative sera (Figure 3).

Generally, true negative and true positive samples defined by a "gold standard" method are very important to calculate the diagnostic specificity and sensitivity of a newly established assay. However, as gold standard is difficult to achieve, relative standards of comparison either from experimentally infected or vaccinated animals is often neces- 
sary [27]. For this purpose, 187 sera from pigs with a known exposure to ASFV infection and 178 sera from pigs that do not have any history of contact to ASFV were used in our experiment. Similarly, a commercially available blocking ELISA kit (Ingezim PPA COMPAC) for ASFV antibody detection were used as a standard evaluating method. The ASFV Ingezim PPA COMPAC is OIE recommended commercially available blocking ELISA [32] and is one of the three commercial ELISA kits in use by the Foreign Animal Disease Diagnostic Laboratory (FADDL) [11]. Initially, serum samples were classified as ASFV-seronegative and ASFV-seropositive based on their origin and their result on Ingezim PPA COMPAC and later all the samples were tested by the newly developed cELISA. Considering the PI value of these 365 pig serum samples, a receiver-operating characteristic (ROC) analysis was used to estimate the accuracy of 2A7-based cELISA. According to the result from ROC curve approach, nearly $100 \%$ diagnostic specificity was obtained, when the cut-off value was set to $25 \%$ but the sensitivity of the test was $86.6 \%$. Thus, we select a cut-off value of $20 \%$, which presents the optimal balance of sensitivity (92.5\%) and specificity $(98.8 \%)$. Also, the result achieved from the newly developed cELISA showed an excellent agreement (kappa value $=0.912)$ with those obtained from Ingezim PPA COMPAC, which indicates a very good concordance between the two methods.

To our knowledge, the two commercially available blocking-ELISA for ASFV antibody detection depends on the use of monoclonal antibodies against p72 (Ingenasa-Ingezim PPA COMPAC K3; Ingenasa, Madrid, Spain) and p30 (ID.vet- blocking ELISA; ID Screen African Swine Fever Competition, Grabels, France). This work identifies the usefulness of p54 monoclonal antibodies on this regard and will extend the previously available cELISAs for the diagnosis of ASFV infection. As a whole, the developed cELISA based on p54 monoclonal antibody has a high diagnostic sensitivity and specificity. Although, further validation using large sample size is strongly recommended, the work presented here encourages the use of p54 monoclonal antibodies in competitive ELISA for ASFV antibody detection.

\section{Materials and Methods}

\subsection{Serum Samples}

A panel of 365 serum samples were analysed with the established 2A7-based competitive ELISA, including 178 negative sera and 187 ASFV positive sera. The origin of samples was:

(A) 178 samples without ASFV exposure: out of these 148 field samples were collected in 2017, before the outbreak of ASFV in China and were confirmed to be negative by the commercial ASFV antibody detection kit, while the other 30 negative sera were originated from specific pathogen free (SPF) pigs.

(B) 187 samples with ASFV exposure: all ASFV positive samples used in this study were obtained from pigs experimentally inoculated either with the wild type Pig/HLJ/2018 ASFV isolate or with the recently developed seven-gene deleted ASFV vaccine candidate HLJ/18-7GD [33]. Those samples were collected at different time of interval between the year 2019 to 2020 in our laboratory and their status was determined by the commercial ASFV antibody detection kit.

(C) Standard control: hyperimmune serum from pig immunized with p54 recombinant protein was used as a positive control.

(D) Heterologous positive sera: to evaluate the specificity of the assay, four known positive sera for other porcine pathogens, namely, porcine reproductive and respiratory syndrome (PRRS), highly pathogenic porcine reproductive and respiratory syndrome (HPPRRSV), Classical swine fever virus (CSFV) and Pseudorabies virus (PrV) were tested. All anti-sera were detected and certified by Harbin Guo sheng Biological Testing Technology Co., Ltd, Harbin, China.

\subsection{Constructing $p 54$ Recombinant DNA}

P54/E183L (excluding the predicted transmembrane domain) based on African swine fever virus isolate Pig/HLJ/2018 (accession. No. MK333180.1) [34,35] was amplified using 
a forward primer TCTTCAAGAAAGAAAAAAGCTGCTG and a reverse TTACAAGGAGTTTTCTAGGTCTTTATGCGT. Amplicon were inserted downstream from the malE gene of E. coli into the vector pMAL-c5x using NdeI and EcoRI restrictions sites introduced into the sense and antisense primers, respectively. Briefly, polymerase chain reaction (PCR) amplified E183L/p54gene (399bp) and pMAL-c5x vector were digested with NdeI and EcoRI restriction enzymes and accordingly ligated with T4 DNA ligase. Recombinant gene (pMAL-c5x-54) were then transformed to DH5 $\alpha$ E. coli competent cells and incubated over night at $37^{\circ} \mathrm{C}$ in an agar plate with ampicillin. On the next day, perfection of the correct insert was checked by PCR and positive samples were confirmed by DNA sequencing (Comate Bioscience Co., Ltd, Jilin, China).

\subsection{Expression and Purification of Recombinant ASFV-p54 Protein}

Recombinant E183L/p54 gene of ASFV isolate Pig/HLJ/2018 was expressed as maltose binding protein -tagged (MBP-tagged) fusion protein in Escheria coli ER2523 strain (made competent in our laboratory according to a protocol by Chung [36]). Expression was facilitated by adding $0.5 \mathrm{mM}$ isopropyl- $\beta$-D-1-thiogalactoside (IPTG) and successful expression was examined by sodium dodecyl sulfate-polyacrylamide gel electrophoresis (SDS-PAGE) analysis of cell lysates. To purify p54 recombinant protein, bacterial cells were harvested by centrifugation, resuspended in pre-cold PBS $(25 \mathrm{~mL} /$ liter of bacterial culture) and lysed by sonication. After centrifugation at 12,000 rpm for 30 minutes, supernatants were collected and filtered through $0.22 \mu \mathrm{m}$ and purified using pre-packed MBP Trap column, operated by AKTA AVANT liquid chromatography system. Buffers for purification were prepared according to the manufacturers guide (binding buffer $=20 \mathrm{mM}$ Tris- $\mathrm{HCl}$ and elution buffer $10 \mathrm{mM}$ maltose in binding buffer).

\subsection{P54 Monoclonal Antibody Production}

To produce anti-p54 monoclonal antibodies the standard protocols for hybridoma technology was followed [37]. Female mice (BALB/c), 4-6 weeks age, were immunized by intraperitoneal injection with $100 \mu \mathrm{g}$ of purified p54 recombinant protein, which was emulsified with an equal amount of Freund's complete adjuvant (Sigma, Lot \# SLBZ9888, St. Louis, MO, USA). A conventional immunization protocol was followed on three mice, to select the mouse best responding to p54 antigen. Two booster immunization was done at two-week intervals mixed with incomplete adjuvant. 10 days later from the second boost, blood was taken by tail bleeding and serum antibody titration for p54 was determined. Mouse that presented the highest antibody titration by indirect ELISA was chosen for the final boost without adjuvant and subsequently was sacrificed three days later to harvest spleen cells.

Hybridoma were obtained by fusing SP2/0 mouse myeloma cells with spleen cells by using poly ethyl glycol (PEG) (Sigma, Lot \# RNBF1747, St. Louis, MO, USA). Fused spleen and SP2/0 cells were resuspended with RPMI-1640 medium (Gibco, Lot \# 8120219, ThermoFisher Biomedical products Co., Ltd, Beijing, China) supplemented with $20 \%$ FBS, $1 \%$ antibiotic (penicillin and streptomycin), $0.2 \%$ glutamine and $50 \times$ hypoxanthineaminopterin-thymidine (HAT) (Sigma, Lot \# SLBZ5521, St. Louis, MO, USA). Thereafter, fused cells were dispensed to 96 well micro culture plate $(100 \mu \mathrm{L} /$ well $)$, added to previously prepared feeder cells and incubated under $5 \% \mathrm{CO}_{2}$ at $37{ }^{\circ} \mathrm{C}$. Feeder cells used were BALB/c macrophages, harvested from healthy BALB/c mice by peritoneal wash with $20 \%$ FBS-RPMI medium, distributed in to four 96 well micro culture plate and incubated for 24 hours prior to fusion. After five days of culture, growth medium was replaced with medium containing 50x HAT and on day seven medium containing only 50x hypoxanthinethymidine (Sigma, Lot \# SLBX7957, St. Louis, MO, USA), was used to replace the growth medium in order to reduce the effect of aminopterin. Cell supernatants were assayed 10 days post cell fusion and wells with confluent hybridomas were screened by indirect ELISA using p54 recombinant protein as a coating antigen. subsequently, positive colonies were selected and sub-cloned by limiting dilution to obtain a single hybrid cell per well. 


\subsection{Western Blot Analysis}

The reactivity of monoclonal antibodies to $\mathrm{p} 54$ recombinant protein was examined by western blot analysis. Purified MBP-tagged p54 recombinant protein was separated in a $12 \%$ SDS-PAGE by electrophoresis and separated proteins were transferred in to PVDF membrane. The membrane was then blocked for 1 hour with 5\% skimmed milk. Primary antibodies were added and incubated for $2 \mathrm{hrs}$ at room temperature with a slow agitation. The primary antibodies used were, five anti-p54 monoclonal antibodies (undiluted hybridoma supernatants), anti-MBP monoclonal antibodies diluted as 1:10,000, polyclonal mice sera from mice immunized with recombinant p54 protein diluted 1:400. After extensive washing with PBST, HRP-labelled goat anti-mouse IgG was added and left at room temperature for 1 hour. Finally, protein bands were visualized by digital imaging system after three-time wash with PBST.

\subsection{Indirect ELISA}

For the screening of antibody secreting hybridoma cells, standard protocol for indirect enzyme-linked immunosorbent assay was performed [38]. Briefly, ninety-six-well microtiter ELISA plates were coated with $0.2 \mu \mathrm{g}$ recombinant p54 protein per well (coating concentration was determined by checkboard titration) and incubated overnight at $4{ }^{\circ} \mathrm{C}$. The plate was washed five times with PBST $(0.05 \%$ Tween in PBS, $v / v)$ and plate was blocked with $5 \%$ skimmed milk in PBS, for $1 \mathrm{~h}$ at $37^{\circ} \mathrm{C}$. After washing the plates as above, $50 \mu \mathrm{L}$ undiluted hybridoma supernatants were added. Positive sera from mice immunized with p54 recombinant protein and negative sera from unimmunized mice, diluted 1:200 was also included in duplicate as a control. The plate was incubated for two hours at $37^{\circ} \mathrm{C}$ and washing step was repeated. Thereafter, horse radish peroxidase (HRP) conjugated goat anti-mouse IgG diluted 1:10,000 was added and incubated for $1 \mathrm{~h}$ at $37^{\circ} \mathrm{C}$. Following extensive washing, reaction was developed by adding chromogenic substrate solution (TMB) for 10 minutes and stopped with $2 \mathrm{M}$ sulphuric acid. Result was read at $\mathrm{OD}_{450}$ absorbance.

\subsection{Indirect Immunofluorescent Assay (IFA)}

To evaluate the specificity and ability of anti-p54 monoclonal antibodies to detect ASFV infected cells, IFA was performed on HEK293T cells transfected with p54 recombinant gene and ASFV infected PAM cells. HEK293T cells were grown in 24-well plate at a density of $1.25 \times 10^{5}$ /well in a complete DMEM medium. After 24 hours, cells were transfected with p54 plasmid DNA and incubated at $37^{\circ} \mathrm{C}$ in $5 \% \mathrm{CO}_{2} .24$ hours post transfection, HEK293T cells were washed with PBS and fixed with $4 \%$ formaldehyde for 10 minutes at room temperature. Following washing with PBS, $0.25 \%$ triton was added to each well in order to increase the permeability of the cells and was left at room temperature for 10 minutes. The cells were then incubated with anti-p54 monoclonal antibodies for 1 hour at $37^{\circ} \mathrm{C}$, followed with FICT conjugated goat ant-mouse IgG. After each incubation step, the wells were washed four times with PBS. Finally, result was observed using a fluorescent microscope.

IFA was also done on ASFV infected peripheral alveolar macrophage cells (PAM cells). The general procedure was same with IFA done on HEK 293T cells, except PAM cells were grown in complete RPMI medium supplemented with $10 \%$ porcine serum and were infected with the lethal dose of ASF virus (Pig/HLJ/2018 isolate).

\subsection{Establishment of Monoclonal Antibody Based-Competitive ELISA for African Swine Fever Antibody Detection}

Monoclonal antibodies based-competitive ELISA was performed to assess the potential application of p54 monoclonal antibodies for ASFV diagnosis. The general procedures followed was as follows; ELISA plates were coated with $0.2 \mu \mathrm{g} /$ well p54 antigen and incubated over night at $4{ }^{\circ} \mathrm{C}$. The optimal coating concentration of recombinant protein and dilution of monoclonal antibodies (mAbs) was determined by checkboard titration. On the next day, plates were washed five times with PBST, blocked with $200 \mu \mathrm{L} /$ well 
$5 \%$ skimmed milk and incubated for 1 hour at $37^{\circ} \mathrm{C}$. Following five times wash, plates were either used directly or kept in $-20^{\circ} \mathrm{C}$ for further use. To optimize serum dilution, a serial two-fold dilution (1:10, 1:20, 1:40, 1:80, 1:160, 1:320, 1:640 and 1:1280) were tested against a fixed monoclonal antibody dilution. Later, each well of p54 antigen coated plates were incubated with $50 \mu \mathrm{L}$ pig serum diluted with PBS for 30 minutes at $37^{\circ} \mathrm{C}$. An equal amount of p54 monoclonal antibodies ( $50 \mu \mathrm{L} /$ well) diluted in PBS was then added to each well without being washed. Controls consisting of wells without $\mathrm{mAb}$ or pig serum and wells having only $\mathrm{mAb}$ were included. Serum and $\mathrm{mAb}$ mixture were incubated for $1 \mathrm{hr}$ at $37^{\circ} \mathrm{C}$. Washing step was repeated and HRP conjugated goat anti-mouse IgG (ZSGB-Bio, China, catalog \# ZB-5305) was added and incubated for 1 hour at $37^{\circ} \mathrm{C}$. After extensive washing, $50 \mu \mathrm{L}$ color substrate (TMB) was added to each well and left at room temperature for 15 minutes and reaction was terminated by adding $50 \mu \mathrm{L} 2 \mathrm{M}$ sulphuric acid to each well. Finally, result was read at optical density of $450 \mathrm{~nm}$. The raw data was transformed to an excel spread sheet and accordingly the percent of inhibition (PI value) of each test sample was calculated using the formula: PI $(\%)=\left[\left(\mathrm{OD}_{450}\right.\right.$ value of negative controls $\mathrm{OD}_{450}$ value of sample)/OD 450 value of negative controls] $\times 100 \%$, as described by Wang and his colleagues [39].

\subsection{Data Analysis}

Using the commercial blocking ELISA kit as a standard evaluating method, sensitivity and specificity of the established cELISA was calculated by the web-based MedCalc statistical software (https:/ / www.medcalc.org/calc/diagnostictest.php (accessed on 7 February 2021)). Receiver operating characteristic (ROC) analysis and degree of agreement (kappa value) were analysed using SPSS software for windows, version 26.0 (IBM, Armonk, NY, USA). Similarly, Interactive dot plot diagram was performed via Graph pad prism version 8.0.2. (GraphPad Software Inc. La Jolla, CA, USA) and difference were considered statistically significant when $p$ value was less than 0.05 .

\section{Conclusions}

This study prepared five monoclonal antibodies against the structural p54 protein of ASFV and their diagnostic application were investigated. Through the aforementioned experiments and analysis, we conclude that the newly developed mAb 2A7 based cELISA method offers a promising approach for a rapid and convenient ASFV serodiagnosis, which could be used as alternative to other serological assays for screening possible ASFV infection.

Author Contributions: Conceptualization, Z.B. and D.Z.; Formal analysis, W.T. and L.W.; Investigation, W.T., L.W., G.T., Y.T.H., Z.Z., J.Z., H.H., F.X., F.L. and E.S.; Methodology, F.L. and E.S.; Project administration, Z.B. and D.Z.; Supervision, Z.B. and D.Z.; Writing-original draft, W.T. and L.W.; Writing-review \& editing, Z.B. and D.Z. All authors have read and agreed to the published version of the manuscript.

Funding: This work was supported by the National Key R\&D Program of China (2018YFC1200601), Applied Technology Research and Development Project of Heilongjiang Province (GA19B301), KeyArea Research and Development Program of Guangdong Province (2019B020211004) and the grant from the State Key Laboratory of Veterinary Biotechnology Program (SKLVBP201801).

Institutional Review Board Statement: All procedures involving animals were carried out in strict accordance with recommendation in the guide for the care and use of laboratory animals of the ministry of science and technology of the People's Republic of China. The protocol was approved by the committee on the ethics of animal experiments of the Harbin Veterinary Research Institute (HVRI) of the Chinese Academy of Agricultural Sciences (CAAS).

Informed Consent Statement: Not applicable.

Data Availability Statement: Data sharing is not applicable to this article.

Conflicts of Interest: The authors declare no conflict of interest. 


\section{References}

1. Wang, N.; Zhao, D.; Wang, X.; Wang, J.; Zhang, Y.; Wang, M.; Gao, Y.; Li, F.; Wang, J.; Bu, Z.; et al. Architecture of African swine fever virus and implications for viral assembly. Science 2019, 366, 640-644. [CrossRef]

2. Salas, M.L.; Andrés, G. African swine fever virus morphogenesis. Virus Res. 2013, 173, 29-41. [CrossRef]

3. Blome, S.; Gabriel, C.; Beer, M. Pathogenesis of African swine fever in domestic pigs and European wild boar. Virus Res. 2013, 173, 122-130. [CrossRef] [PubMed]

4. Salguero, F.J. Comparative Pathology and Pathogenesis of African Swine Fever Infection in Swine. Front. Vet. Sci. 2020, 7, 282. [CrossRef] [PubMed]

5. Sánchez-Cordón, P.J.; Núñez, A.; Neimanis, A.S.; Wikström-Lassa, E.; Montoya, M.; Crooke, H.; Gavier-Widén, D. African Swine Fever: Disease Dynamics in Wild Boar Experimentally Infected with ASFV Isolates Belonging to Genotype I and II. Viruses 2019, 11, 852. [CrossRef]

6. Sánchez-Cordón, P.J.; Montoya, M.; Reis, A.L.; Dixon, L.K. African swine fever: A re-emerging viral disease threatening the global pig industry. Vet. J. 2018, 233, 41-48. [CrossRef]

7. Zhou, X.; Li, N.; Luo, Y.; Liu, Y.; Miao, F.; Chen, T.; Zhang, S.; Cao, P.; Li, X.; Tian, K.; et al. Emergence of African Swine Fever in China. Transbound. Emerg. Dis. 2018, 65, 1482-1484. [CrossRef] [PubMed]

8. Netherton, C.L.; Goatley, L.C.; Reis, A.L.; Portugal, R.; Nash, R.H.; Morgan, S.B.; Gault, L.; Nieto, R.; Norlin, V.; Gallardo, C.; et al. Identification and Immunogenicity of African Swine Fever Virus Antigens. Front. Immunol. 2019, 10, 1318. [CrossRef] [PubMed]

9. OIE. Global Situation of African Swine Fever. Report No. 47:2016-2020. Available online: https://rr-asia.oie.int/en/projects/ asf/situational-updates-of-asf-in-asia-and-the-pacific/ (accessed on 15 December 2020).

10. Gallardo, C.; Blanco, E.; Rodriguez, J.M.; Carrascosa, A.L.; Sánchez-Vizcaíno, J.M. Antigenic Properties and Diagnostic Potential of African Swine Fever Virus Protein pp62 Expressed in Insect Cells. J. Clin. Microbiol. 2006, 44, 950-956. [CrossRef] [PubMed]

11. Gallardo, C.; Reis, A.L.; Kalema-Zikusoka, G.; Malta, J.; Soler, A.; Blanco, E.; Parkhouse, R.M.E.; Leitão, A.B. Recombinant Antigen Targets for Serodiagnosis of African Swine Fever. Clin. Vaccine Immunol. 2009, 16, 1012-1020. [CrossRef] [PubMed]

12. Perez-Filgueira, D.M.; Camacho, F.G.; Gallardo, C.; Resino-Talavan, P.; Blanco, E.; Gomez-Casado, E.; Alonso, C.; Escribano, J.M. Optimization and Validation of Recombinant Serological Tests for African Swine Fever Diagnosis Based on Detection of the p30 Protein Produced in Trichoplusia ni Larvae. J. Clin. Microbiol. 2006, 44, 3114-3121. [CrossRef] [PubMed]

13. Cubillos, C.; Gómez-Sebastian, S.; Moreno, N.; Nuñez, M.C.; Mulumba-Mfumu, L.K.; Quembo, C.J.; Heath, L.; Etter, E.M.; Jori, F.; Escribano, J.M.; et al. African swine fever virus serodiagnosis: A general review with a focus on the analyses of African serum samples. Virus Res. 2013, 173, 159-167. [CrossRef] [PubMed]

14. Oviedo, J.; Rodriguez, F.; Gómez-Puertas, P.; Brun, A.; Gómez, N.; Alonso, C.; Escribano, J. High level expression of the major antigenic African swine fever virus proteins p54 and p30 in baculovirus and their potential use as diagnostic reagents. J. Virol. Methods 1997, 64, 27-35. [CrossRef]

15. Barderas, M.G.; Rodríguez, F.; Gómez-Puertas, P.; Avilés, M.; Beitia, F.; Alonso, C.; Escribano, J.M. Antigenic and immunogenic properties of a chimera of two immunodominant African swine fever virus proteins. Arch. Virol. 2001, 146, 1681-1691. [CrossRef]

16. Rodriguez, F.; Alcaraz, C.; Eiras, A.; Yáñez, R.J.; Rodriguez, J.M.; Alonso, C.; Escribano, J.M. Characterization and molecular basis of heterogeneity of the African swine fever virus envelope protein p54. J. Virol. 1994, 68, 7244-7252. [CrossRef]

17. Alcaraz, C.; Brun, A.; Ruiz-Gonzalvo, F.; Escribano, J. Cell culture propagation modifies the African swine fever virus replication phenotype in macrophages and generates viral subpopulations differing in protein p54. Virus Res. 1992, 23, 173-182. [CrossRef]

18. Gómez-Puertas, P.; Rodríguez, F.; Oviedo, J.M.; Brun, A.; Alonso, C.; Escribano, J.M. The African Swine Fever Virus Proteins p54 and p30 Are Involved in Two Distinct Steps of Virus Attachment and Both Contribute to the Antibody-Mediated Protective Immune Response. Virology 1998, 243, 461-471. [CrossRef]

19. Rodriguez, F.; Ley, V.; Gómez-Puertas, P.; García, R.; Rodriguez, J.; Escribano, J. The structural protein p54 is essential for African swine fever virus viability. Virus Res. 1996, 40, 161-167. [CrossRef]

20. Rodríguez, J.M.; García-Escudero, R.; Salas, M.L.; Andrés, G. African Swine Fever Virus Structural Protein p54 Is Essential for the Recruitment of Envelope Precursors to Assembly Sites. J. Virol. 2004, 78, 4299-4313. [CrossRef] [PubMed]

21. Alonso, C.; Miskin, J.; Hernáez, B.; Fernandez-Zapatero, P.; Soto, L.; Canto, C.; Rodríguez-Crespo, I.; Dixon, L.; Escribano, J.M. African Swine Fever Virus Protein p54 Interacts with the Microtubular Motor Complex through Direct Binding to Light-Chain Dynein. J. Virol. 2001, 75, 9819-9827. [CrossRef] [PubMed]

22. García-Mayoral, M.F.; Rodríguez-Crespo, I.; Bruix, M. Structural models of DYNLL1 with interacting partners: African swine fever virus protein p54 and postsynaptic scaffolding protein gephyrin. FEBS Lett. 2011, 585, 53-57. [CrossRef] [PubMed]

23. Kollnberger, S.; Gutierrez-Castañeda, B.; Foster-Cuevas, M.; Corteyn, A.; Parkhouse, R.M.E. Identification of the principal serological immunodeterminants of African swine fever virus by screening a virus cDNA library with antibody. J. Gen. Virol. 2002, 83, 1331-1342. [CrossRef] [PubMed]

24. Reis, A.L.; Parkhouse, R.M.E.; Penedos, A.R.; Martins, C.; Leitão, A.B. Systematic analysis of longitudinal serological responses of pigs infected experimentally with African swine fever virus. J. Gen. Virol. 2007, 88, 2426-2434. [CrossRef]

25. Lokhandwala, S.; Waghela, S.D.; Bray, J.; Martin, C.L.; Sangewar, N.; Charendoff, C.; Shetti, R.; Ashley, C.; Chen, C.-H.; Berghman, L.R.; et al. Induction of Robust Immune Responses in Swine by Using a Cocktail of Adenovirus-Vectored African Swine Fever Virus Antigens. Clin. Vaccine Immunol. 2016, 23, 888-900. [CrossRef] 
26. Alcaraz, C.; Rodriguez, F.; Oviedo, J.; Eiras, A.; De Diego, M.; Alonso, C.; Escribano, J. Highly specific confirmatory Western blot test for African swine fever virus antibody detection using the recombinant virus protein p54. J. Virol. Methods 1995, 52, 111-119. [CrossRef]

27. Jacobson, R. Validation of serological assays for diagnosis of infectious diseases. Rev. Sci. Tech. 1998, 17, 469-526. [CrossRef]

28. Alejo, A.; Matamoros, T.; Guerra, M.; Andrés, G. A Proteomic Atlas of the African Swine Fever Virus Particle. J. Virol. 2018, 92. [CrossRef]

29. Teklue, T.; Sun, Y.; Abid, M.; Luo, Y.; Qiu, H. Current status and evolving approaches to African swine fever vaccine development. Transbound. Emerg. Dis. 2020, 67, 529-542. [CrossRef]

30. OIE. African Swine Fever. In OIE Manual of Diagnostic Tests and Vaccines for Terrestrial Animals; World Organization for Animal Health (OIE): Paris, France, 2012; pp. 1067-1081.

31. Sánchez-Vizcaíno, J.M.; Mur, L.; Gómez-Villamandos, J.C.; Carrasco, L. An Update on the Epidemiology and Pathology of African Swine Fever. J. Comp. Pathol. 2015, 152, 9-21. [CrossRef]

32. Bergeron, H.C.; Glas, P.S.; Schumann, K.R. Diagnostic specificity of the African swine fever virus antibody detection enzymelinked immunosorbent assay in feral and domestic pigs in the United States. Transbound. Emerg. Dis. 2017, 64, 1665-1668. [CrossRef]

33. Chen, W.; Zhao, D.; He, X.; Liu, R.; Wang, Z.; Zhang, X.; Li, F.; Shan, D.; Chen, H.; Zhang, J.; et al. A seven-gene-deleted African swine fever virus is safe and effective as a live attenuated vaccine in pigs. Sci. China Life Sci. 2020, 63, 623-634. [CrossRef]

34. Zhao, D.; Liu, R.; Zhang, X.; Li, F.; Wang, J.; Zhang, J.; Liu, X.; Wang, L.; Zhang, J.; Wu, X.; et al. Replication and virulence in pigs of the first African swine fever virus isolated in China. Emerg. Microbes Infect. 2019, 8, 438-447. [CrossRef]

35. Wen, X.; He, X.; Zhang, X.; Zhang, X.; Liu, L.; Guan, Y.; Zhang, Y.; Bu, Z. Genome sequences derived from pig and dried blood pig feed samples provide important insights into the transmission of Africa swine fever virus in China in 2018. Emerg. Microbes Infect. 2019, 8, 303-306. [CrossRef] [PubMed]

36. Chung, C.T.; Niemela, S.L.; Miller, R.H. One-step preparation of competent Escherichia coli: Transformation and storage of bacterial cells in the same solution. Proc. Natl. Acad. Sci. USA 1989, 86, 2172-2175. [CrossRef] [PubMed]

37. Groth, S.D.S.; Scheidegger, D. Production of monoclonal antibodies: Strategy and tactics. J. Immunol. Methods 1980, 35, 1-21. [CrossRef]

38. Hnasko, R. Methods in Molecular Biology. In ELISA; Humana Press: New York, NY, USA, 2015; Volume 1318, pp. 51-59.

39. Wang, L.; Mi, S.; Gong, W.; Shi, J.; Madera, R.; Ganges, L.; Borca, M.V.; Ren, J.; Cunningham, C.; Cino-Ozuna, A.G.; et al. A neutralizing monoclonal antibody-based competitive ELISA for classical swine fever C-strain post-vaccination monitoring. BMC Vet. Res. 2020, 16, 14. [CrossRef] 